DEBQ emotional eating score, EDE-Q eating concern score, EDE- shape concern score, EDE-Q weight concern score, EDE-Q total score and CDI score $(>19) \quad(p$ : 0,03 ; 0,02 ; 0,$007 ; 0,01 ; 0,049$ respectively). No significant association was found between BED and depression (CDI score >19). At Pearson's correlation test, positive correlations were found between DEBQ emotional eating score, DEBQ total score, EDE-Q eating concern score, EDE-Q shape concern score, EDE-Q weight concern score, EDE-Q total score and the STAİ score $(\mathrm{p}=0.0001)$. No significant association was found between BED and the STAI scores.

Conclusion In obese adolescents, disordered eating attitudes and behaviors could be associated with anxiety and depressive symptoms. Thus, all adolescents with obesity should be screened for their eating attitudes and behaviors and also for their emotional health.

\section{P531 PREVALENCE OF OBESITY AMONG SCHOOLCHILDREN IN ST. PETERSBURG}

Vera Gricinskaya, Valeria Novikova*, Margarita Gurova. St. Petersburg State Pediatric University, St. Petersburg, Russian Federation

\subsection{6/archdischild-2019-epa.866}

Introduction The upward steady trend in the prevalence of obesity in the pediatric population during the last decades is a significant medical and social problem.

Objectives To study the prevalence of obesity among schoolchildren in St. Petersburg.

Methods Totally 6449 schoolchildren aged 6-17 years old had been enrolled in the cross-sectional study with the use of random sampling techniques. All investigated children were subdivided into 4 subgroup: I - 946 children 6-7 years old; II - 2092 children aged 8-11 years; III - 2925 children aged 12-15 лет; IV - 486 children aged 16-17 years old. We measured the length and weight of the body, waist circumference (WC), calculated body mass index (BMI). We diagnosed obesity if BMI value was above 95 percentile in accordance with the standards centile scale 'WHO Growth Reference 2007'. A visceral obesity was diagnosed when the WC exceeded more than 90 percentile according to the scale proposed by International Diabetes Federation (2007). Data was analyzed using the statistical package «STATISTICA v.7.0».

Results Obesity was diagnosed in $13.8 \%$ of schoolchildren. We have identified the following relationships between age, gender, and obesity. According to the gender, obesity was found to be more common among boys $(15.8 \%)$ than girls $(11.5 \%$; $\mathrm{p}<0.001)$. The difference is statistically significant in the I $(\mathrm{p}<0.001)$, II $(\mathrm{p}<0.001)$ and IV $(\mathrm{p}=0.01)$ groups. In the third age group, the number of boys and girls with obesity was equal.

According to the age, boys with obesity in IV gr. were less than in I gr. $(16.8 \%$; p $=0.004)$, II gr. $(17.4 \% ; \mathrm{p}=0.001)$ and in III gr. $(15.6 \% ; p=0.007)$. Similarly, obese girls in IV gr. were less $(3.6 \%)$ than in I gr. $(9.3 \%$; p $=0.004)$, in II gr. (9.5\%; $\mathrm{p}=0.002)$ and especially in III gr. (15.1\%); p < $0.001)$. The largest number of obese girls were detected in III group, compare to II and I groups ( $\mathrm{p}<0.001$ ).

Waist circumference exceeding 90 percentile are defined in $2.7 \%$ of schoolchildren. The number of boys was greater $(3.7 \%)$ than girls $(1.7 \% ; \mathrm{p}=0.009)$.
Conclusion We found a high prevalence of obesity among schoolchildren of the megalopolis - up to $13.8 \%$.

\section{P532 USE OF NEW TECHNOLOGIES FOR THE FOLLOW-UP IN ADOLESCENT OBESITY; MOBILE HEALTH INTERVENTION (MHI) A RANDOMIZED CONTROLLED TRIAL}

Myriam Dabbas*, Geraldine Lepage, Emilie Boedoz, Adrien Charrat, Marion Consfroy, Yann Manh. Hôpital Necker Enfants Malades, AP-HP, Paris, France

\subsection{6/archdischild-2019-epa.867}

Childhood obesity continues to be a key challenge in France. Our unit is facing in high demand with limited resources.

The purpose of this study is to evaluate the efficacy of distance follow-up on decreasing of body mass index compared to traditional management in obese adolescents.

Methods After a traditional intensive period consisting in a weekly family-based multidisciplinary intervention for two months, patients are randomized in the two arms, traditional (group 1) or distance follow-up (group 2).

Traditional follow up based on face-to-face multidisciplinary consultations every three months.

The distance monitoring based on a mobile application dedicated to food behaviour change and physical activity, with weekly self-monitoring' goal setting, physical activity and healthy eating support' and monthly weight assessment, moreover pre-programmed feedbacks 'motivational strategies' are included.

Only anonymous data were collected After one year of follow up, adolescents were evaluated for weight loss, compliance and quality of life, the two groups were compared.

All adolescents (more than 11 years) attending our unit for obesity care, were invited to be include the trial. Exclusion criteria were mental disability, syndromic obesity, bariatric surgery or no French speaking.

Results 91 adolescents (42 boys) were enrolled in this trial,

79 patients were randomized (14\% loss of follow-up during the intensive care).

Mean age was $12.82 \pm 1.3$ years, mean of Body Mass Index (BMI) was $30.8 \pm 4.08$ corresponding to $3.94 \pm 0.7$, Z- score BMI.

The loss of follow up at one year was $9 \%$ in the two groups (after 4 months on average).

The group ' 1 ' had a mean of 4 hours/patient/year of face to face visits while the group'2' had a mean of 0.5 hour/ patient/year of distant chat.

After one year of follow-up the success rate (decreasing of BMI Z- score more than 0.5 points) was $21 \%$ in the group 1 and $30 \%$ in the group 2, 66.7\% and 65\% of adolescents stabilized their BMI Z-score in the two groups respectively.

Obesity is a chronic disease that needs a strong and long term follow-up; the addition of new technologies for distant monitoring can be helpful to promote healthy lifestyle in obese adolescents and can support them to have more autonomy and fewer appointments with professionals.

Nevertheless, despite an intensive program of care with a multidisciplinary dedicated team, the impact on weight loss still modest, and our future efforts must focus on improving individual results. 